\title{
Revelación de información voluntaria sobre capital intelectual: factores explicativos en empresas cotizantes argentinas $^{*}$
}

\section{Voluntary disclosure of information on Intellectual Capital: explanatory factors in Argentine listed companies}

\author{
Gabriela Analía García \\ Cecilia Rita Ficco ${ }^{2}$ \\ Gustavo Sander ${ }^{3}$
}

\section{Resumen}

Para identificar características de las empresas que pueden resultar significativas para explicar la política de revelación de información voluntaria sobre los activos intangibles incorporados en los componentes del capital intelectual, se ha realizado un estudio de tipo correlacional que apunta a analizar la relación entre ciertas características de las empresas cotizantes en el Mercado de Valores de Buenos Aires y un índice que recoge la información que voluntariamente revelan estas empresas sobre los componentes de dichos elementos intangibles. El análisis de regresión múltiple aplicado indica que son las empresas con altos niveles de endeudamiento las que parecen estar más predispuestas a brindar información adicional sobre los componentes del capital intelectual, en tanto que las más rentables parecen oponerse a este tipo de revelación.

\section{Palabras clave}

Información voluntaria, capital intelectual, características empresariales.

\section{Códigos de clasificación JEL: M41- C35}

\section{Abstract}

This investigation was made in order to identify those characteristics of companies that could be significant in explaining the voluntary disclosure policy about Intangible Assets incorporated into the components of Intellectual Capital. In this way, a correlational study has been conducted in order to analyze the relationship between certain characteristics of the companies listed on the Stock Exchange of Buenos Aires and an index that collects the information voluntarily revealed by these companies about the components of Intellectual Capital. The multiple regression analysis applied indicates that companies with high debt levels seem to be more willing to provide additional information about the components of Intellectual Capital, while the most profitable ones seem to oppose to such disclosure.

\section{Keywords}

Voluntary disclosure, intellectual capital, enterprise features.

De la investigación: El enfoque de la utilidad de la información financiera para la toma de decisiones aplicada a intangibles de empresas cotizantes, no cotizantes y del sector público aprobada en la Universidad Nacional de Río Cuarto, Argentina.

1 Contador Público. Docente e Investigadora de la Universidad Nacional de Río Cuarto y la Universidad Nacional de Villa María - Argentina. Correo electrónico: ggarcia@fce.unrc.edu.ar

2 Magíster en Sistemas de Información para la toma de decisiones. Docente Investigadora de la Universidad Nacional de Río Cuarto y la Universidad Nacional de Villa María, Argentina. Correo Electrónico: cficco@eco. unrc.edu.ar

3 Magíster en Sistemas de Información para la toma de decisiones. Docente Investigador de las Universidad Nacional de Río Cuarto y la Universidad Nacional de Villa María, Argentina. Correo Electrónico: gsader@eco. unrc.edu.ar 


\section{Introducción}

Los mercados de capitales se caracterizan por una situación de asimetría de información, en la que los insiders (directivos, accionistas de referencia, entre otros) disponen de más información sobre la situación y perspectivas futuras de la empresa, que los inversores externos no vinculados a la gestión, lo que determina que el funcionamiento de los mercados de capitales dependa en gran medida de la cantidad y calidad de la información específica difundida por las sociedades que operan en el mercado (Gómez, Iñiguez \& Poveda, 2005).

Debido a ello, las empresas que cotizan en los mercados de capitales han incrementado la revelación voluntaria de información como complemento de la información obligatoria exigida por las normas vigentes (Cañibano \& Sánchez, 2004; Ramírez, 2010; Ficco, Sader \& Bersía, 2012).

Un tipo de información que resulta relevante para la valoración de las empresas por parte de los inversores externos, es la referida al conjunto de intangibles vinculados al conocimiento, reconocido como capital intelectual, respecto del cual es prácticamente nula la información complementaria requerida por las normas contables, por tratarse de elementos intangibles que no cuentan con los atributos que permiten asignarles mediciones contables confiables (Sader, Ficco, Tissera \& García, 2013).

De hecho, las empresas que operan en los mercados de capitales actualmente divulgan crecientes cantidades de información voluntaria sobre sus intangibles, debido a que, tal como indican Cañibano \& Sánchez (2004), necesitan ser transparentes y dignas de credibilidad, por la poca confianza que los inversores parecen tener en la fiabilidad de la información financiera y en el funcionamiento de los mercados financieros.

Muchos estudios han tratado de buscar explicaciones a las diferencias de comportamiento en la revelación de información por parte de las empresas, fundamentalmente de aquella divulgada de forma voluntaria. En estas explicaciones, el tamaño de la empresa, su rentabilidad, el sector en el que la empresa realiza su actividad, la cotización bursátil, la concentración del accionariado, la calidad de la firma de auditoría, el nivel de endeudamiento y la cantidad de activos intangibles son algunas de las variables que de alguna manera se han asociado con la política de revelación de información (Sader, Ficco, Tissera \& García, 2013). La relación entre estos factores y la política de revelación de información ha sido abordada desde distintas teorías, principalmente, desde la teoría de la agencia, de la señal, de los costos políticos y de los costos del propietario.

En este marco, y tomando como partida la siguiente pregunta de investigación: ¿qué es lo que motiva a las empresas que operan en los mercados de capitales a la revelación voluntaria de información sobre su capital intelectual?, es que hemos 
realizado diferentes estudios ${ }^{4}$ en vista a identificar el tipo de información voluntaria sobre activos intangibles revelada por las empresas que cotizan en el Mercado de Valores argentino, así como también, la cantidad de la misma, habiéndose elaborado, con tales propósitos, un índice de revelación de ese tipo de información. Complementariamente, se ha avanzado en el análisis de aquellas características propias de las empresas cotizantes argentinas que pueden resultar significativas para explicar la política de revelación de información voluntaria sobre los activos intangibles identificados en los componentes del capital intelectual. Son precisamente los resultados de estos estudios, que no han arrojado conclusiones contundentes, los que constituyen la motivación de este trabajo, el cual pretende avanzar en el análisis y en la interpretación de los resultados previamente obtenidos para profundizar el estudio de la problemática planteada.

A tal fin, se ha realizado un estudio de tipo correlacional que apunta a analizar la relación entre ciertas características de las empresas cotizantes en el Mercado de Valores de Buenos Aires y la revelación de información que realizan sobre sus activos intangibles vinculados al conocimiento. Específicamente, se ha aplicado un análisis de regresión múltiple para establecer la influencia relativa de distintas variables, representativas de las características antes referidas, en la cantidad de información contable que suministran las empresas, la cual se ha medido a través del índice de revelación voluntaria de información sobre capital intelectual construido.

El diseño de investigación utilizado es de tipo no experimental y, en relación con la dimensión temporal, se aplicó un diseño trasversal, ya que los datos se han recolectado para un momento dado del tiempo.

En esta línea, tras esta introducción, el trabajo ha sido dividido en cuatro secciones. En la primera se expone el marco teórico y los antecedentes empíricos del trabajo. En la segunda, se muestra la metodología aplicada para el desarrollo de la investigación realizada en el entorno del mercado de capitales argentino y, en la tercera sección, se presentan los resultados de esta y su discusión. En la última sección se plantean las conclusiones.

4 En el marco del Programa de Investigación "El enfoque de la utilidad de la información financiera para la toma de decisiones aplicada a intangibles de empresas cotizantes, no cotizantes y del sector público", aprobado y financiado por la Universidad Nacional de Río Cuarto en el marco del Programa Nacional de Incentivos, para el período 2012-2015. 


\section{Marco teórico y antecedentes empíricos}

\section{Factores explicativos de la revelación de información sobre los componentes del capital intelectual desde la teoría y la empírica}

En busca de respuestas a la pregunta inicial respecto de qué es lo que motiva a las empresas que operan en los mercados de capitales a la revelación voluntaria de información sobre su capital intelectual, hemos iniciado un recorrido por la bibliografía que recoge resultados de investigación empírica sobre la temática en diferentes países. Así, hemos detectado que entre los factores que más frecuentemente han sido asociados a una política activa de revelación de información financiera, se encuentran el tamaño de la empresa, su rentabilidad, el nivel de endeudamiento, la cuota de mercado, la concentración del accionariado, el ramo de actividad, la cotización bursátil, el prestigio de la firma de auditoría, la cobertura de analistas y la cantidad de activos intangibles.

Pero lo cierto es que, si bien en la mayoría de los casos los fundamentos teóricos respecto de la elección de las características explicativas es coincidente, la evidencia empírica respecto del comportamiento de estas variables y su relación con el grado de divulgación de información, no ha arrojado conclusiones contundentes y, a menudo, ha brindado resultados contradictorios (Sader, Ficco, Tissera \& García, 2013). Es decir, tal como indican García \& Sánchez (2006), para la mayor parte de las variables analizadas existe una amplia inestabilidad en los resultados, por lo que no ha podido arribarse hasta el momento a una teoría general que permita explicar o predecir el comportamiento de las empresas en relación con la política de revelación de información, sino a un conjunto de teorías parciales que han sido utilizadas para explicar evidencias empíricas, a menudo contradictorias, sobre la cantidad de información divulgada.

Entre esas teorías se encuentran la Teoría de la Agencia, la Teoría de la Señal y la Teoría de los Costos Políticos, de acuerdo con las cuales la divulgación voluntaria de información por parte de las empresas se puede considerar, no solo como un instrumento para reducir posibles conflictos de intereses, sino también, como un mecanismo para señalar al mercado la mejor posición relativa de una empresa frente a las demás (García \& Sánchez, 2006), generando un impacto en su valor.

La teoría de la empresa (Jensen \& Meckling, 1976) entiende a la empresa como un nexo de contratos entre agentes y principales, donde el conflicto de intereses entre ellos y el costo que tales implican, vienen a explicar el rol de la información contable en la definición y control del cumplimiento de los contratos, y con ello, la reducción de los costos de agencia. Y la teoría de los procesos políticos, que parte de la premisa que los políticos llevarán a cabo acciones tendientes a generar una trasferencia de fondos desde las empresas hacia ellos. En este contexto, los datos contables serían utilizados para elaborar y justificar regulaciones a la actividad empresarial, como 
impuestos, subsidios, regulación de precios, monopolios, normas de calidad. Así, la revelación puede ser una herramienta que permita reducir los costos políticos derivados de la transferencia de riquezas al sector público.

Otro de los desarrollos teóricos que permite explicar la política de información financiera es la teoría de la señal (Giner, 1995, 1997). Señalar al mercado aquellos aspectos que pueden tener el atributo de reducir las asimetrías de información que se dan entre la empresa y los inversionistas, puede motivar la revelación de información, buscando un efecto en la valoración de las acciones.

De este modo, es posible afirmar que, también, la revelación de información sobre capital intelectual puede estar motivada en la reducción de los costos de agencia, de los costos políticos y de los derivados de las asimetrías de información. Particularmente, en las asimetrías en relación con los inversores, ya que tienen efecto en la valoración de la empresa, por lo que la política de revelación de información financiera puede ser entendida como un intento de reducir costos y crear valor.

Sin embargo, y habiendo profundizado en el desarrollo de los aspectos teóricos desde los que es posible justificar políticas más proactivas para la revelación voluntaria de información sobre los componentes del capital intelectual, es necesario hacer espacio a otros argumentos respecto de las causas que pueden limitar este tipo de revelación, vinculadas a los denominados costos del propietario (Rodríguez, 2004). Dentro de ellos se agrupan los costos derivados de las desventajas competitivas que pueden generar el dar a conocer a los competidores este tipo de información, y los costos de recolección y procesamiento de esa información (Gray \& Roberts, 1993; Babío et al., 2001). Muchos autores priorizan a los primeros como limitación a la revelación voluntaria de información sobre los componentes del capital intelectual, por cuanto los costos de recolección y procesamiento ya fueron asumidos por los propietarios de las empresas para elaborar información para la gestión interna de la firma; en tanto, frente a la ausencia de obligación de brindar información sobre los componentes del capital intelectual, y debido a su fuerte vinculación con las causas de ventajas competitivas de las empresas, se considera a los costos derivados de las desventajas competitivas como los más importantes condicionantes para este tipo de publicación (Edwards \& Smith, 1996).

Otra cuestión que es importante tener en cuenta, se relaciona con el efecto moderador que muy posiblemente tenga el grado de desarrollo del mercado de capitales y el nivel de incertidumbre que puede verificarse en los distintos países. Estudios al respecto han demostrado un mayor grado de divulgación de información voluntaria en los países cuyos mercados de capitales tienen un mayor desarrollo (Sader, Ficco, Tissera \& García, 2013).

En lo que respecta a los trabajos empíricos que se han realizado con el objetivo de explicar las políticas de revelación de información, el tamaño es el factor explicativo que más se repite. 
La teoría predice la existencia de una asociación positiva entre el nivel de revelación de información y el tamaño de la empresa por varias razones. Desde la teoría de la agencia las empresas de mayor tamaño establecen más contratos y de mayor complejidad que las más pequeñas, por lo que el incentivo a una actitud proactiva de revelación viene dado por la necesidad de reducir los altos costos de agencia; a su vez, estarán más expuestas a litigios, cuyas consecuencias serán más importantes cuanto mayor sea la compañía (Skinner, 1994), y una mayor revelación pretenderá evitarlos o prevenirlos (Kasznik \& Lev, 1995).

Desde la teoría de la señal, en empresas más grandes, las asimetrías de información entre directores e inversores serán mayores y podrán devenir en problemas de selección adversa. Una mayor divulgación estará motivada en evitar esta situación a través de la emisión de señales, o sea, divulgando información privada; ya que ciertamente las empresas grandes están sometidas a mayores demandas de información por parte de los inversores y analistas, debido a su alto grado de complejidad. Una mayor comunicación ayudará a los participantes del mercado a comprender mejor sus actividades y resultados (Eaton \& Rosen, 1983).

Por otro lado, es razonable suponer que las empresas más grandes se tornan más visibles, y por tanto, sujetas a más intervención y costos políticos que el resto; donde la revelación puede resultar un buen intento de los directivos para reducir dichos costos y minimizar la transferencia de riquezas hacia el sector público.

Esta relación positiva entre revelación de información y tamaño ha sido demostrada en numerosos estudios, tanto vinculados a la revelación de información voluntaria (Cerf, 1961; Buzby, 1975; Kahl \& Belkaoui, 1981; McNally, Eng \& Hasseldine, 1982; Chow \& Wong-Boren, 1987; Lang \& Lundholm, 1993; García \& Monterrey, 1993; Rodríguez, 2004, Herrera, 2013), como obligatoria (Wallace, Naser \& Mora, 1994), o una combinación de ambas (Cooke, 1989, 1992; Giner, 1997).

Sin embargo, a pesar de que desde la teoría se ofrecen numerosos argumentos respecto de esta relación positiva, y gran cantidad de trabajos empíricos demuestran esta relación, lo cierto es que en países en los que los mercados de capitales se encuentran escasamente desarrollados, como Argentina, el tamaño puede no asociarse positivamente a la cantidad de información sobre capital intelectual que revelan las empresas. Se puede suponer que el beneficio revelado sigue siendo la principal fuente de información absorbida por el usuario colectivo y, como las grandes empresas son aquellas que generalmente muestran mayores resultados revelados, por ende, no necesitan revelar otro tipo de información para alentar expectativas al usuario (Ficco, García \& Sader, 2013).

Respecto del resto de las variables que tradicionalmente han sido analizadas a fin de indagar acerca de su poder explicativo según la revelación de información financiera, la teoría resulta contundente, pero no así los resultados empíricos. 
Así, en relación con la rentabilidad, una mayor rentabilidad y margen se constituye en una señal de buenas noticias, en un indicador de calidad de la gestión, que lleva a los agentes a revelar mayor cantidad de información para mejorar sus condiciones contractuales (Lang \& Lundholm, 1993; Rodríguez Pérez, 2004), garantizando mejores remuneraciones.

Desde la teoría del proceso político cabe suponer que la empresa facilitará más información con el fin de justificar sus elevados beneficios (Giner, 1995; Cooke, 1989, Wallace et al., 1994) y evitar obligaciones legales (Lang \& Lundholm, 1993). Sin embargo, argumentos legales, también sugieren que las empresas con peores resultados podrían estar motivadas a revelar mayor información para evitar posibles demandas judiciales (Skinner, 1994).

Por último, desde la teoría de la señal, las empresas con alta rentabilidad pueden desear dar información adicional al mercado, señalando su buena situación, en búsqueda de un impacto positivo en el precio de las acciones.

No obstante, desde la perspectiva de los costos del propietario, es posible considerar que aquellas empresas más rentables estén menos predispuestas a brindar información adicional sobre los componentes del capital intelectual, como modo de evitar dar a conocer a sus competidores información sobre sus fuentes de ventajas competitivas (Rodríguez, 2004).

Los resultados de las investigaciones empíricas respecto de la relación entre revelación de información y rentabilidad no son consistentes. Algunos trabajos muestran la existencia de una correlación positiva (Cerf, 1961; Singhvi \& Desay, 1971; Gray \& Roberts, 1993; Lang \& Lundholm, 1993), mientras que otros no la detectan (McNally et al., 1982; García \& Monterrey, 1993; Wallace et al., 1994; Raffournier, 1995; Giner, 1997).

La política financiera de la empresa, en particular su estructura de capital, y la necesidad de obtener financiación externa adicional, inciden notablemente en la decisión de revelación voluntaria de información (Gómez, Iñiguez \& Poveda, 2005), pero el efecto del endeudamiento en la revelación puede ser tanto positivo como negativo.

La Teoría de la Agencia afirma que se espera una mayor transferencia de riqueza de los acreedores a los accionistas conforme el nivel de endeudamiento sea mayor, ya que los costos de agencia son más altos para empresas que utilizan más financiación ajena. En este sentido, sería de esperar una relación positiva entre la divulgación de información voluntaria y el nivel de endeudamiento (García \& Sánchez, 2006), ya que una mayor revelación voluntaria puede contribuir a reducir los costos de agencia de la deuda, facilitando que los acreedores valoren mejor la capacidad de la compañía para cumplir sus obligaciones (Gómez; Iñiguez \& Poveda, 2005). En igual sentido, Rodríguez Pérez (2004) sostiene que un elevado endeudamiento conduce a una mayor proactividad en la revelación de información solo cuando esta es voluntaria. 
Sin embargo, la evidencia empírica al respecto no es concluyente, Raffournier (1995) y Lang \& Lundholm (1993) encuentran una relación positiva entre revelación voluntaria y endeudamiento; no siendo apoyada esta asociación en la mayor parte de los estudios sobre el tema, que evidencian una ausencia de relación o una asociación significativa, pero de signo contrario al esperado (Chow \& Wong-Boren, 1987; McKinnon \& Dalimunthe, 1993; Hossain et al., 1994; Meek et al., 1995; Ho \& Wong, 2001, y Ferguson et al., 2002).

Algunos autores como Healey \& Palepu (1995) y Baiman \& Verrechia (1996) justifican estos resultados argumentando que los acuerdos financieros pueden disminuir el incentivo de los directivos para publicar información voluntaria. Por otra parte, de acuerdo con Adrem (1999) y Larrán (2001), estos resultados pueden ser parcialmente explicados por una hipótesis alternativa propuesta por Jensen (1986), consistente en la asociación negativa existente entre la cantidad de flujos libres de tesorería y los costos de agencia. Según este autor, el endeudamiento puede ser entendido como un control externo que garantiza que los directivos están actuando de acuerdo con los intereses de sus accionistas, siendo la función de control del endeudamiento más eficiente en aquellas empresas que generen mucha tesorería y no tengan muchas oportunidades de crecimiento. Así, bajo el argumento de los flujos libres de tesorería la relación entre endeudamiento y cantidad de información divulgada puede entenderse como negativa.

La revelación de información varía según el sector en el que la empresa desarrolle su actividad. Es de esperar que empresas del mismo sector enviarán señales similares, ya que si alguna de ellas no lo hace, puede ser interpretado por el mercado como que se ocultan malas noticias (Rodríguez, 2004).

Así mismo, se supone que los costos políticos pueden depender de la industria en que se sitúe la empresa (Watts \& Zimmerman, 1986). También los costos de propietarios son similares entre sectores, puesto que tanto las desventajas competitivas como los costos de elaboración y preparación son diferentes entre industrias. Por ello, se cree que las empresas situadas en el mismo sector de actividad tendrán prácticas y niveles de revelación de información similares. Esta hipótesis es contrastada en diversos estudios. Algunos la confirman (Cooke, 1992; Gray \& Roberts, 1993; McKinnon \& Dalimunthe, 1993; Mitchell et al., 1995) pero otros no (McNally et al., 1982; Wallace et al., 1994; Giner, 1997).

Estudios como el desarrollado por Rodríguez (2004) muestran que las diferencias entre sectores resultan significativas. Siendo el sector servicio el que mayor cantidad de información revela y el manufacturero el que menos, principalmente, en lo relacionado con la revelación sobre fuentes de ventajas competitivas responsables de la creación de valor en la empresa.

Los estudios sobre la relación entre el crecimiento de la empresa y la revelación de información han arrojado también resultados contradictorios. La relación 
anterior es confirmada por García \& Monterrey (1993) utilizando las ventas como variable para medir el crecimiento, pero no así con el crecimiento del activo total. Rodríguez (2004) encuentra que el crecimiento de las ventas es un factor explicativo de la revelación de información sobre fuentes de ventaja competitiva, pero solo en lo relacionado con las capacidades de la empresa. Sin embargo, los resultados de McNally, Eng \& Hasseldine (1982) no apoyan la relación entre crecimiento y revelación de información, utilizando diferentes variables para medir el crecimiento.

Finalmente, con respecto a la cantidad de activos intangibles reconocidos contablemente, las empresas con más intangibles tienen una mayor asimetría informativa respecto de los inversores, por lo que se espera que hagan más revelación pública. Los beneficios se espera que provengan de una reducción de los costos de agencia derivados de dicha asimetría, dada la relación positiva existente entre intangibles (valor de las oportunidades de crecimiento) y costos de agencia (Smith \& Watts, 1992; Gaver \& Gaver, 1993). Así mismo, la revelación sobre intangibles ayuda a los inversores y acreedores a entender la inversión realizada y valorar la empresa.

Según Gómez, Iñiguez \& Poveda (2005, p. 16), "resulta lógico pensar que es posible que sean las empresas que tienen mayores intangibles no reconocidos en balance las que proporcionen mejor información".

\section{Estudios previos y resultados para el mercado de capitales argentino}

En el marco de las investigaciones desarrolladas por el equipo de investigación que integran los autores de este trabajo, se realizó un primer estudio, de tipo exploratorio, para iniciar el análisis de la relación entre ciertas características de las empresas cotizantes en el Mercado de Valores de Buenos Aires y la revelación de información sobre sus activos vinculados al conocimiento. Para estudiar a las empresas cotizantes en el Mercado de Valores de Buenos Aires se tomó como muestra el conjunto de empresas líderes cotizantes en dicho mercado, por ser estas las que concentran la mayor cantidad de negocios; quedando la muestra conformada por las 19 empresas que se mantuvieron en la categoría de líderes durante el período 2008-2012 .

En lo relativo a las técnicas de recolección de datos, se utilizaron aquellos públicamente disponibles, y fueron tomados de los estados contables anuales consolidados publicados por las empresas que integran el Índice Merval 25 y que presentaron para los ejercicios económicos cerrados en 2012.

5 Las empresas líderes son las que integran el Índice Merval 25 y el período 2008-2012 es el considerado para desarrollar la investigación correspondiente al Programa de Investigación "El enfoque de la utilidad de la información financiera para la toma de decisiones aplicada a intangibles de empresas cotizantes, no cotizantes y del sector público" en el cual se enmarca el presente trabajo. 
Para examinar empíricamente la relación entre la información que revelan voluntariamente las empresas sobre su capital intelectual y sus características, se seleccionaron, en principio, aquellas que más frecuentemente han sido estudiadas: el ramo de actividad, el tamaño de la empresa y su crecimiento, su rentabilidad, el nivel de endeudamiento y la cantidad de activos intangibles reconocidos. Así mismo, se definieron variables relevantes para medir esas características. Así, el tamaño, se aproximó computando dos medidas alternativas: el activo total y las ventas. Como medida de rentabilidad se ha utilizado la rentabilidad sobre ventas y también, la rotación del activo. El endeudamiento se ha medido a través del ratio de endeudamiento. Y la cantidad de activos intangibles reconocidos a través del ratio entre activos intangibles y activo total. Finalmente, para medir el crecimiento de las empresas se ha tomado tanto el crecimiento de los activos como el de las ventas, que fueron calculados con base en los datos contenidos en los estados contables anuales consolidados correspondientes al período 2008-2012.

Por otra parte, se definió un índice de revelación voluntaria de información sobre capital intelectual, basado en los elementos de información no obligatoria que dejan ver las empresas sujetas a estudio. Estos elementos fueron obtenidos a partir del análisis de la memoria y de la información complementaria a los estados financieros ${ }^{6}$ y se agruparon en las tres categorías básicas en que habitualmente se asocian los componentes del capital intelectual.

Para el análisis de estos datos en este primer estudio, de tipo exploratorio, se aplicó un análisis de componentes principales (ACP) y, se complementó con un análisis clúster.

El objetivo planteado se centró en comparar las distintas empresas sujetas a estudio de acuerdo con la relevancia que tiene la información sobre activos intangibles vinculados al conocimiento y a detectar, si existen, grupos de empresas que se caractericen por la actividad desarrollada, su tamaño, rentabilidad, endeudamiento, cantidad de activos intangibles reconocidos y crecimiento, y que puedan formarse de acuerdo con la importancia que en ellos pueda tener la revelación de información sobre dichos activos.

Este estudio inicial arrojó resultados que se encuentran publicados en Ficco, García \& Sader (2013), dentro de los cuales merecen destacarse los siguientes:

- Se detectaron, por un lado, correlaciones positivas elevadas entre las variables: Activo Total, Ventas, Activos Intangibles/Activo Total y Crecimiento del Activo y, por otro, correlaciones también elevadas, pero negativas, entre

6 Es de destacar que si bien la memoria y la información complementaria que acompaña a los estados financieros son de presentación obligatoria, contienen información que las empresas revelan voluntariamente, en la que se encuentra la relativa a los intangibles vinculados al conocimiento. Fundamentalmente, es la memoria anual el informe en el cual las empresas presentan información cualitativa y no financiera sobre estos intangibles. 
dichas variables y la que mide la Revelación de Información sobre Capital Intelectual.

Las correlaciones positivas elevadas muestran que las empresas que poseen un activo total más elevado también presentan un monto de ventas más elevado, un mayor crecimiento de su activo y los activos intangibles que reconocen contablemente representan una parte más significativa del activo total con respecto a las demás empresas.

Las correlaciones importantes, pero negativas, antes referidas indican que las empresas de mayor tamaño, con mayor crecimiento de su activo y con mayor cantidad de intangibles reconocidos respecto de su activo total, son las que menos información revelan voluntariamente sobre su capital intelectual.

- Así mismo, analizando las correlaciones de las variables activas con los distintos factores, surge un primer factor que opone, claramente, las empresas de mayor tamaño y crecimiento y con mayor cantidad de intangibles reconocido, contra las que revelan más información voluntaria sobre Capital Intelectual ${ }^{7}$.

$\mathrm{Y}$, al analizar estos resultados, queda claro que el tamaño, el crecimiento de las empresas y la cantidad de intangibles reconocidos se oponen a la revelación voluntaria de información sobre capital intelectual, de modo que las empresas de mayor tamaño, las que más han crecido y las que reconocen contablemente una mayor cantidad de intangibles son las que revelan menor cantidad de información sobre su capital intelectual.

- La pertenencia a un sector no resultó determinante de la cantidad de información revelada sobre intangibles vinculados al conocimiento, mientras que la rentabilidad y el endeudamiento tampoco aparecieron como factores explicativos del nivel de revelación de ese tipo de información.

- El análisis clúster, aplicado para examinar si existen grupos de empresas que presenten valores semejantes en las variables medidas, mostró agrupamientos de empresas que, en ningún caso, se realizaron teniendo en cuenta el nivel de revelación de información sobre su capital intelectual. Las clases se formaron reuniendo en cada una de ellas empresas que son semejantes en relación con otras características.

$7 \quad$ En el segundo factor, en cambio, se opone a las empresas con mayor rentabilidad y crecimiento en cuanto a las ventas contra las empresas de menor rentabilidad y crecimiento en la misma variable. Pero este factor no aporta información relevante para el objetivo planteado en este análisis. 


\section{Materiales y métodos}

Los resultados obtenidos a partir del análisis inicial y exploratorio que se han reseñado hasta el momento, plantearon la necesidad de avanzar sobre la temática, al proponer el desarrollo de una nueva investigación que arroje resultados que permitan profundizar el análisis de los factores que influyen en la revelación voluntaria de información sobre capital intelectual que realizan las empresas que operan en el mercado de capitales argentino.

Para ello se avanzó en la aplicación de otro método estadístico para el análisis de datos: el análisis de regresión, lo que implicó, así mismo, una reconsideración sobre las variables cuantitativas utilizadas en el estudio anterior, tal como se explica seguidamente.

En lo que respecta a las fuentes de datos, al igual que en el estudio exploratorio antes referido, se utilizaron datos contables de las empresas estudiadas públicamente disponibles. Dichos datos pudieron obtenerse de los estados financieros que las empresas presentan en la Bolsa de Comercio de Buenos Aires. Específicamente, los datos fueron tomados de los estados contables anuales consolidados ${ }^{8}$ publicados por las empresas que presentaron dichos estados para ejercicios económicos cerrados en $2012^{9}$.

Para el estudio de las empresas cotizantes en el mercado de capitales argentino se tomó como muestra el conjunto de empresas líderes cotizantes en el Mercado de Valores de Buenos Aires, por ser estas las que concentran la mayor cantidad de negocios. No obstante, se trabajó solo con las diecinueve empresas que se mantuvieron en esa categoría durante el período de estudio: 2008-2012.

En lo que respecta a las variables utilizadas, al progresar en el análisis de la base de datos, han surgido una serie de limitaciones que condujeron a una segunda selección de las variables explicativas del modelo. Así, se ha seleccionado una única variable para cada uno de los factores estudiados. Esta elección se fundamenta en los altos niveles de correlación existentes entre ellas. A su vez se ha descartado la variable cualitativa ramo de actividad, debido a la carencia de relevancia detectada en los análisis previos, lo cual permite centrar el análisis en el comportamiento de variables cuantitativas. También se han eliminado las variables indicadoras del factor crecimiento, ya que abarcan un período pre y pos (Normas Internacionales de Información Financiera - NIIF), siendo estas las limitaciones más relevantes detectadas para avanzar en el análisis comparativo de los resultados obtenidos en sendos estudios realizados.

8 Es importante resaltar que se han utilizado los estados contables consolidados para las empresas que han presentado este tipo de información. En el caso de las empresas que solo han presentado información individual, esta es la que se ha utilizado en el estudio.

9 Se tomaron datos para 2012 a los efectos de poder comparar los resultados de este estudio con el realizado previamente. 
De este modo, los factores que se proponen como explicativos del índice de revelación de información sobre el capital intelectual, son: el tamaño, el nivel de endeudamiento, la rentabilidad sobre ventas y la cantidad de intangibles reconocidos como activos. Y las variables utilizadas para medirlos son, respectivamente, el logaritmo neperiano del activo total, el ratio de endeudamiento, la rentabilidad sobre ventas y el ratio activos intangibles sobre activo total.

Finalmente, para el análisis estadístico de los datos se ha aplicado el modelo de regresión lineal múltiple, cuya ecuación se detalla seguidamente con las referencias correspondientes para la identificación de las variables utilizadas:

$$
\operatorname{IRICI}_{i=} \beta_{0}+\beta_{1} x \mathrm{LNAT}_{i}+\beta_{2} x \mathrm{REND}_{i}+\beta_{3} x \mathrm{RVTAS}_{i}+\beta_{4} x R A I_{i}+\varepsilon_{i}
$$

Siendo, para cada empresa $i$ :

$I R I C I=$ Índice de Revelación de Información sobre Capital Intelectual.

$L N A T=$ Logaritmo Neperiano (LN) del Activo Total, como variable indicativa del tamaño de la empresa.

$R E N D=$ Ratio de Endeudamiento, como variable indicativa del nivel de endeudamiento.

$R V T A S=$ Rentabilidad sobre Ventas, como variable indicativa de la rentabilidad.

$R A I=$ Ratio de Activos Intangibles, como variable indicativa de la cantidad de activos intangibles.

El modelo presenta, así, como variable dependiente, al Índice de Revelación de Información sobre Capital Intelectual y, como variables explicativas o independientes, al logaritmo neperiano del activo total, al ratio de endeudamiento, a la rentabilidad sobre ventas y al ratio activos intangibles sobre activo total, las que permiten medir ciertas características seleccionadas de las empresas sujetas a estudio, como son: el tamaño, el nivel de endeudamiento, la rentabilidad sobre ventas y la cantidad de intangibles reconocidos como activos, respectivamente.

\section{Resultados y discusión}

Todas las variables incorporadas al modelo presentan una distribución normal, condición necesaria para poder correr la regresión. Es debido a ello que se ha utilizado como indicador del tamaño el logaritmo neperiano del activo total.

La tabla 1 presenta los resultados de la regresión multivariante del IRICI respecto de las variables explicativas consideradas. El modelo tiene una capacidad explicativa aceptable. En conjunto, las variables independientes explican aproximadamente el $36 \%$ de la varianza de la variable dependiente. 
Tabla 1. Resultados del Modelo de Regresión Múltiple

\begin{tabular}{|l|c|c|c|c|c|}
\hline $\begin{array}{c}\text { Coeficiente de } \\
\text { correlación múltiple }\end{array}$ & $\mathbf{R}^{\wedge} \mathbf{2}$ & $\mathbf{R}^{\wedge} \mathbf{2}$ ajustado & Error típico & Observaciones \\
\hline \multicolumn{1}{|c|}{0,708} & 0.501 & 0.358 & 0.050 & 19 \\
\hline & Coeficientes & Error típico & Estadístico t & Probabilidad \\
\hline Constante & 0.431 & 0.176 & 2.450 & 0.028 \\
\hline LNAT & -0.008 & 0.008 & -1.035 & 0.318 \\
\hline RVTAS & -0.119 & 0.048 & -2.474 & 0.027 \\
\hline REND & 0.006 & 0.003 & 2.008 & 0.064 \\
\hline RAI & 0.085 & 0.219 & 0.388 & 0.704 \\
\hline
\end{tabular}

Fuente: Autores

Para el análisis de los resultados obtenidos se ha trabajado con un nivel de significatividad de entre el 0.05 y el 0.1 . Los resultados muestran una relación positiva y significativa $(p<0.1)$ entre el índice de revelación de información sobre capital intelectual (IRICI) y el endeudamiento, y una relación negativa y significativa $(p<0.05)$ entre el IRICI y la rentabilidad. No han resultado determinantes, de la cantidad de información voluntaria sobre activos intangibles revelada por las empresas cotizantes en el mercado de valores argentino, ni el tamaño ni la cantidad de intangibles reconocidos como activos. Si en el modelo se desagregan el ratio de cantidad de activos intangibles reconocidos en relación con el activo total, el ratio referido al valor llave y el ratio de otros intangibles reconocidos, el modelo se mantiene inalterable respecto de los resultados que arroja.

La confrontación de los resultados de esta investigación con otros estudios sobre la misma problemática, permite aportar las siguientes consideraciones:

- En los resultados obtenidos en Ficco, García \& Sader (2013), como se analizó anteriormente en el ACP, tanto el endeudamiento como la rentabilidad, no surgen como variables explicativas de la divulgación de información voluntaria sobre el capital intelectual; por lo que al complementarlo con los resultados de la regresión, sí se puede observar la relación que existe entre estas variables y la divulgación de información voluntaria sobre capital intelectual.

- Adicionalmente, y a la luz del estudio revelado en García \& Sánchez (2006), se puede interpretar la relación negativa entre rentabilidad y divulgación de información voluntaria sobre capital intelectual, en el sentido de que la baja rentabilidad es incentivo a la revelación voluntaria, debiendo ser extensamente justificada para evitar demandas judiciales. 
- Respecto a la relación positiva entre endeudamiento y revelación voluntaria de información sobre capital intelectual, Gómez e Iñiguez \& Poveda (2005) utilizan la teoría de la agencia para explicar ese comportamiento, afirmando que los costos de agencia son más altos para empresas que utilizan más financiación ajena. Por lo que es de esperar que una mayor revelación voluntaria puede contribuir a reducir los costos de agencia de la deuda, facilitando que los acreedores valoren mejor la capacidad de la compañía para cumplir sus obligaciones.

- Una consideración especial merece el comportamiento de la variable vinculada al tamaño de la empresa, ya que la teoría ofrece numerosos argumentos respecto a una relación positiva y significativa entre el tamaño y una política más proactiva de revelación voluntaria, que gran cantidad de trabajos empíricos demuestran. Pero lo cierto es que esa relación no ha podido ser demostrada en este estudio. Si bien es probable que ello se deba a algunas limitaciones, es posible justificar este resultado retomando algunos de los argumentos referidos a que en países en los que los mercados de capitales se encuentran escasamente desarrollados, como es el caso de Argentina, el tamaño puede no resultar significativo debido a la cantidad de información sobre capital intelectual que revelan las empresas. Un argumento en tal sentido se puede encontrar en Ficco, García \& Sader (2013), quienes basados en numerosas investigaciones, suponen que el beneficio revelado sigue siendo la principal fuente de información absorbida por el usuario colectivo $\mathrm{y}$, como las grandes empresas son aquellas que generalmente muestran mayores resultados revelados, no necesitan brindar otro tipo de información para alentar expectativas al usuario.

\section{Conclusiones}

El análisis de regresión múltiple genera resultados que difieren de los obtenidos previamente sobre la misma base de datos. Contrariamente a lo observado, a través del ACP, ni el tamaño ni la cantidad de intangibles activados en relación con el total de activos resultan determinantes de la cantidad de información voluntaria revelada sobre capital intelectual. A su vez, las variables que en aquel análisis no resultaron relevantes, como la rentabilidad y el endeudamiento, sí resultan significativas como regresores del índice de revelación de información sobre capital intelectual.

Si se analizan los resultados obtenidos en la regresión, a la luz del objetivo planteado, examinar empíricamente la relación que existe entre la información sobre capital intelectual que voluntariamente brindan las empresas cotizantes en el mercado de valores argentino, y ciertas características de tales empresas, resulta claro que las empresas más rentables parecen oponerse a este tipo de revelación, por el contrario, las empresas con altos niveles de endeudamiento parecen estar más predispuestas a brindar información adicional sobre los componentes del capital intelectual. 
La relación significativa y negativa entre rentabilidad sobre venta e índice de revelación, resulta contraria a lo que predicen las teorías que vinculan la mayor rentabilidad a una actitud más proactiva a la revelación voluntaria de información sobre su capital intelectual; teorías que explican dicha conducta como modo de enviar señales de buenas noticias al mercado que impacten en el precio de las acciones, así como también, de divulgar las causas de esas rentabilidades altas con el fin de evitar obligaciones legales.

Sin embargo, sería posible relacionar la baja revelación de información voluntaria sobre los componentes del capital intelectual detectada, con el propósito de evitar divulgar las causas de ventajas competitivas que ocasionan esas mayores rentabilidades. Ello, debido a que muy probablemente el peso relativo de los costos del propietario, ocasionados por el acceso de los competidores a esa información superen los beneficios de divulgarla.

Como se ha anticipado, en mercados escasamente desarrollados, como el argentino, el impacto de las señales enviadas al mercado sobre el precio de las acciones resulta incierto, siendo el beneficio revelado la mayor información absorbida por el usuario colectivo.

En esta línea argumental podríamos concluir que en los países cuyos mercados de capitales están poco desarrollados, los costos de divulgación de información privilegiada exceden los beneficios de brindarla, y es debido a ello que el tamaño no resulta determinante con relación a la política de revelación voluntaria que asumen las empresas cotizantes en el mercado de valores argentino. Por lo que solo se justifica brindar información adicional sobre activos intangibles cuando el nivel de endeudamiento es alto o la rentabilidad de la firma es baja.

Para poder avanzar en esta conclusión será necesario continuar este estudio, ampliando la muestra e incorporando al análisis otras variables que han sido excluidas. Si los resultados se mantuviesen será necesario avanzar en una nueva investigación que permita demostrar que la revelación voluntaria de información sobre los componentes del capital intelectual en Argentina se asocia a las malas noticias financieras vinculadas a los bajos niveles de rentabilidad, altos niveles de endeudamiento y retrocesos en la tasa de crecimiento.

\section{Referencias}

Adrem, A. H. (1999). Essays on disclosure practices in Sweden-Causesand Effects. Lund Studies in Economics and Management 51. The Institute of Economic Research, Lund University Press, Sweden.

Babío, M. R., Muiño, M. F., y Vidal, R. (2001). La decisión de publicar información voluntaria. (Un análisis de la opinión de los directores de la empresa española). XI Congreso AECA, septiembre, 2001, Madrid. 
Baiman, S. \& Verrechia, R. R. (1996). The relation among capital markets, financial disclosure, product efficiency and insider trading. Journal of Accounting Research, 34(1), 1-22.

Buzby, S. (1975). Company size, listed versus unlisted stocks, and the extent of financial disclosure. Journal of Accounting Research, spring, pp. 16-37.

Cañibano, L., \& Sánchez, M. (2004). Medición, gestión e información de intangibles: lo más nuevo. Revista de Contabilidad y Dirección, 1, 99-139.

Cerf, A. R. (1961). Corporate Reporting and Investment Decisions. University of California Press.

Chow, C. W. \& Wong-Boren, A. (july, 1987). Voluntary financial disclosure by Mexican Corporations. Accounting Review, pp. 533-541.

Cooke, T. E. (1992). The impact of size, stock market listing and industry type on disclosure in the annual reports of japanese listed corporations. Accounting and Business Research, summer, pp. 229-237.

Cooke, T. E. (july, 1989). Disclosure in the corporate annual reports of Swedish companies. Accounting and Business Research, pp. 533-541.

Eaton, J. \& Rosen, H. (1983). Agency, delayed compensation and the structure of executive Remuneration. Journal of Finance, (38), 1489-1505.

Edward. P. S. y Smithr, A. (1996). Competitived isadvantage and voluntary disclosures: the case of segmental reporting. British Accounting Review, (28), $155-172$.

Ferguson. M., Lam, K., \& Lee, G. (2002). Voluntary disclosure by state-owned enterprises listed on the stock exchange of Hong Kong. Journal of International Financial Management and Accounting, 13(2), 125-152.

Ficco, C., García, G., \& Sader, G. (2013). Aspectos clave en la revelación del capital intelectual en empresas cotizantes del mercado de capitales argentinos. Revista Visión Contable, (11), 330-362. Ed. Unaula. Medellín, Colombia.

Ficco, C., Sader, G., \& Bersia, P. (2012). El valor de la organización y los activos intangibles: información disponible en empresas cotizantes en el mercado de capitales argentino. XIX Jornadas de Intercambio de Conocimientos Científicos y Técnicos. UNRC, Río Cuarto, Argentina.

García B., M. A., \& Monterrey, Mayoral, J. (1993). La revelación voluntaria en las compañías españolas cotizadas en bolsa. Revista Española de Financiación y Contabilidad, (74), 53-70. 
García, E., \& Sánchez, J. (2006). Un estudio meta-analítico de los factores determinantes de la revelación de información. Revista Española de Financiación y Contabilidad, XXXV(132), 761-788.

Gaver, J., y Gaver, K. (1993). Additional evidence on the association between the investment opportunity set and corporate financing, dividend, and compensation policies. Journal of Accounting and Economics, (16), 125-160.

Giner Inchausti, B. (1995). La divulgación de información financiera: una investigación empírica. Instituto de Contabilidad y Auditoría de Cuentas. Madrid.

Giner Inchausti, B. (1997). The influence of company characteristic and accounting regulation on information disclosed by Spanish firms. The European Accounting Review, 6, 45-68.

Gómez, J., Iñiguez, R., Poveda, F. (2005). Revelación voluntaria de información y características de las sociedades cotizadas en el mercado de capitales español. Revista Española de Financiación y Contabilidad, (131), 8-32.

Gray, S. y Roberts, C. (1993). Voluntary information disclosures: the attitudes of UK multinationals. In Grays, J.; Coenenbear, G. G. y Gordopn, D. (Eds.). International group accounting: issues in European harmonisation (2a. ed.). London.

Healey, P., y Palepu, K. (1995). The challenges of investor communications: the case of CUC International. Journal of Financial Economics, 38, 111-141.

Herrera, E. (2013). Factores que explican la extensión de revelación de activos intangibles de los bancos que cotizan en la Bolsa de Valores de Panamá. Contaduría \& Administración, 58(3), 173-202.

Ho, S. S. M. \& Wong, K. S. (2001). A study of the relationship between corporate governance structures and the extent of voluntary disclosure. Journal of International Accounting, Auditing \& Taxation, 10, 139-156.

Hossain, M., Tan, L. M., \& Adams, M. (1994). Voluntary disclosure in an emerging capital market; some empirical evidence from firms listed on the Kuala Lumpur Stock Exchange. The International Journal of Accounting, 29, 334-351.

Jensen, M. (1986). Agency costs of free cash flow, corporate finance and takeovers. American Economic Review, Papers and Proceedings, 76, 323-329.

Jensen, M., \& Mecklinwg, H. (1976). Theory of the firm: managerial behavior, agency costs and ownership structure. Journal of Financial Economics, 3, Issue $1,305-360$.

Kasznik, R. \& Lev, B. (1995). To warn or not to warn: Management disclosures in the face of an earnings surprise. The Accounting Review, (70), 113-134. 
Kahl, A., \& Belkaoui, A. (1981). Bad annual report disclosure adequacy internationally. Accounting and Business Research, (11), 189-196.

Lang, M., \& Lundholm, R. (1993). Cross sectional determinants of analysis ratings of corporate disclosures. Journal of Accounting Research, 31(2), 246-271.

Larrán Jorge, M. (2001). El mercado de información voluntaria en las bolsas de valores. Madrid: ICAC.

Mckinnon, J. L., \& Dalimunthe, L. (1993). Voluntary disclosure of segment information by australian diversified companies. Accounting and Finance, 33(1), $33-50$.

Mcnally, G. M., Eng, L. H., \& Hasseldine, C. R. (1982). Corporate financial reporting in New Zealand: An analysis of user preferences, corporate characteristic and disclosure practices for discretionary information. Accounting and Business Research, winter, pp. 11-20.

Meek, G. K., Roberts, C. B., \& Gray, S. J. (1995). Factors influencing voluntary annual reports disclosures by US and UK and continental European multinational corporations. Journal of International Business Studies, 26, 555-572.

Mitchell, J. D., Chia, C. W. L., \& Loh, A. S. (nov., 1995). Voluntary disclosure of segment information: further Australian evidence. Accounting and Finance, pp. $1-16$.

Raffournier, B. (1995). The determinants of voluntary financial disclosure by Swiss listed companies. The European Accounting Review, 4,(2), 261-280.

Ramírez, Y. (2010). Divulgación de la información sobre intangibles en organizaciones españolas. Contabilidad y Negocios, 9(5), 57-67.

Rodríguez, G. (2004). Factores explicativos de la revelación voluntaria de información. Revista Española de Financiación y Contabilidad, XXXIII(122), 705-739.

Sader, G., Ficco, C., Tissera, P., \& García, G. (2013). Presentación de información voluntaria y valor de la empresa: factores explicativos en empresas cotizantes en el mercado de capitales argentino. XXXIV J.U.C. Universidad Nacional del Litoral, Santa Fe, Argentina.

Skinner, D. (1994). Why firms voluntarily disclosure bad news. Journal of Accounting Research, (32), 38-60.

Smith, C., \& Watts, R. (1992). The investment opportunity set and corporate financing, dividend, and compensation policies. Journal of Financial Economics (32), 263-292. 
Wallace, R. S., Naser, K., \& Mora, A. (1994). The relationship between comprehensiveness of corporate annual reports and firm characteristics in Spain. Accounting and Business Research, 25(97), 41-53.

Watts, R. L., \& Zimmerman, J. L. (1986). Positive accounting theory. Englewood Cliffs, New Jersey: Prentice Hall. 